\title{
Molecular Dynamics Simulations of Small Peptides: Can One Derive Conformational Preferences from ROESY Spectra?
}

\author{
Christine Peter, ${ }^{[\mathrm{a}]}$ Magnus Rueping ${ }^{[\mathrm{b}]}$ Hans Jakob Wörner, ${ }^{[\mathrm{a}]}$ Bernhard Jaun, ${ }^{[\mathrm{b}]}$ \\ Dieter Seebach, ${ }^{[b]}$ and Wilfred F. van Gunsteren ${ }^{*[a]}$
}

\begin{abstract}
Folding properties of $\beta$-peptides were investigated by means of NMR experiments and MD simulations of $\beta$-dipeptides, which serve as small test systems to study the influence of stereocenters and side chains on hydrogen-bond and consequently on secondary-structure formation. Two stereoisomers, $S R$ and $S S$, of a Val-Phe dipeptide, and of the corresponding AlaAla dipeptide, and a Gly-Gly dipeptide were simulated in methanol for $40 \mathrm{~ns}$. In agreement with experiment, the isomers of the Val-Phe dipeptide adopt quite different conformers at $298 \mathrm{~K}$,
\end{abstract}

the differences being reduced at $340 \mathrm{~K}$. Interestingly, the $S R$ isomer shows enhanced hydrogen bonding at the higher temperature. The adopted conformations are primarily determined by the $R$ or $S$ side chain substitution, and less by the type of side chain. Back-calculation of ${ }^{1} \mathrm{H}$ ROESY spectra and ${ }^{3} J$ coupling constants from the MD simulations and comparison with the experi-

Keywords: $\beta$-peptides • conformation analysis - molecular dynamics . NMR spectroscopy $\cdot$ peptides mental data for the Val-Phe dipeptides shows good agreement between simulation and experiment, and reveals possible problems and pitfalls, when deriving structural properties of a small and extremely flexible molecule from NMR data only. Inclusion of all aspects of internal dynamics is essential to the correct prediction of the NMR spectra of these small molecules. Cross comparison of calculated with experimental spectra for both isomers shows that only a few out of many ROESY peaks reflect the sizeable conformational differences between the isomers at $298 \mathrm{~K}$.

\section{Introduction}

$\beta$-Peptides belong to the group of so called foldamers, ${ }^{[1]}$ that is, non-natural oligomeric compounds with a strong tendency to adopt specific, three-dimensional conformations. They exhibit secondary structure elements that are very similar to the ones found in $\alpha$-peptides and proteins, namely they are able to form intramolecular hydrogen bonds to generate various left- and right-handed helices $\left(3_{14}, 2.5_{12}\right.$ and 10/12 helices), turns and sheets. ${ }^{[2,3]}$ Due to this feature and the fact that the secondary structure elements found are very stable already for rather short oligomers, these systems are ideal to study folding properties, that is both the propensities of specific sequences for special secondary structure elements and the process of folding. ${ }^{[1,4]}$ Especially for helical structures, the helix type is closely related to a specific hydrogen-bond-

[a] Prof. W. F. van Gunsteren, C. Peter, H. J. Wörner Laboratorium für Physikalische Chemie ETH-Hönggerberg, HCI, 8093 Zürich (Switzerland) Fax: $(+41) 1-632-1039$

E-mail:wfvgn@igc.phys.chem.ethz.ch

[b] M. Rueping, Prof. B. Jaun, Prof. D. Seebach Laboratorium für Organische Chemie ETH Zürich, 8093 Zürich (Switzerland) ing pattern. The relative stability of the helix types depends on the substitution pattern, that is, the types of the amino acid side chains, the substitution pattern, the backbone carbon atom ( $\alpha$ or $\beta$ ) to which the side chain is attached, and the stereochemistry at this backbone carbon atom. The shortest possible $\beta$-peptide that can still form the hydrogen bonds that are characteristic for the various helix types, is a dimer, which is, therefore, the ideal test system to systematically analyze the influence of the three mentioned factors on the hydrogen-bond and consequently on the secondarystructure formation.

NMR and circular dichroism (CD) spectroscopy are the commonly used experimental tools for structure determination of peptides. They can be optimally complemented by molecular dynamics (MD) simulations, which not only provide an atomic resolution picture to compare with but also insight into dynamical processes, which can be essential for a correct interpretation of the experimental data. Simulation studies of several $\beta$-peptides which adopt different secondary structures have already proven that it is possible to correctly reproduce the experimentally predicted structure by unbiased MD simulations and to obtain a well-sampled folding/unfolding equilibrium. ${ }^{[5-7]}$ As simulations provide both structural and dynamical information they also reveal possible problems when interpreting experimental data of pepti- 
des which are-compared to proteins-small and highly flexible compounds. The experimental structure analysis is by no means trivial if the underlying ensemble cannot be represented by a single conformation but rather by an equilibrium of possibly even very different structures. ${ }^{[8,9]}$ In our study, we will focus on the influence of internal dynamics and conformational equilibria on NMR, particularly on ${ }^{1} \mathrm{H}$ ROESY, spectra of short $\beta$-peptides. We will point out, why the experimental tools that were developed for the structural and dynamical analysis of proteins have to be applied with caution as soon as it comes to smaller systems. There are several methods to analyze the dynamics of proteins based on NMR relaxation data ${ }^{[10,11]}$ namely the so called model free approach, ${ }^{[12]}$ spectral-density mapping ${ }^{[13]}$ or approaches that fit the experimental data to analytical motional models. ${ }^{[14,15]}$ Common to most of these approaches is the fact, that they consider a relatively large molecule where the overall rotational motion is slow compared with the internal motion and where overall and internal dynamics are completely decoupled. Another way to analyze NMR relaxation data is by comparison with MD simulations. For this, it is important to understand the relationship between the interproton distances, or more precisely the time series of interproton vectors, which can be obtained from an MD simulation, and the relaxation rates which finally determine the intensities in the spectrum. The individual cross-relaxation rates belonging to each pair of protons are determined through the spectral densities, that is, the Fourier coefficients of the time correlation function which represents the reorientational motion of the corresponding interproton vector. Detailed derivations can be found in the literature. ${ }^{[16,11]}$ These relaxation rates describe the relaxation of the system of spins through a set of coupled differential equations, which can be solved by diagonalization of the socalled relaxation matrix. ${ }^{[17-19]}$ There are several ways how internal dynamics contribute to the relaxation rates:

- Fluctuationsin the interproton distances.

- The individual effective correlation times of the motions of the interproton vectors contain contributions from both tumbling motions and internal dynamics and are thus different for each proton pair.

- Due to the angular or orientational averaging in the time correlation functions and due to superposition of overall rotation and possibly various types of internal motions, the individual time correlation functions can have different (non-monoexponential) functional forms.

Besides these three factors, another contribution to the final intensities results from spin-diffusion, that is, indirect cross-relaxation through a more than pairwise interaction of spins, which is only taken into account by solving the coupled relaxation-matrix equation. Not doing this is equivalent to assuming that the intensity of each peak linearly depends on the corresponding cross-relaxation rate, which is correct in the limit of a zero-mixing time. For this reason, this is called the initial-rate approximation. ${ }^{[20]}$

When comparing NMR relaxation data (namely NOESY or ROESY intensities) with results from MD simu- lations, various levels of accuracy can be applied. The crudest approach is to compare distance averages obtained from the simulation with experimentally derived distances. The appropriate averaging method $\left(\left\langle r^{-6}\right\rangle\right.$ or $\left\langle r^{-3}\right\rangle$ averaging) depends on the system size and the relation between the timescales of rotational motion and internal fluctuations. ${ }^{[21]}$ This method assumes that any other contribution to the individual cross-relaxation rates is identical for all proton pairs. The next, more accurate approach is to explicitly compute the time correlation functions of all or only of selected proton pairs and to obtain the corresponding spectral densities either by numerical Fourier transformation or analytically after fitting the correlation functions to a set of exponential functions. This has been done for proteins, ${ }^{[22,23]}$ where often only the correlation functions representing the internal dynamics are computed, and for lipid membranes. ${ }^{[2]}$ This method additionally accounts for individual correlation times and individual functional forms of the correlation functions but not for spin diffusion. The separation into overall and internal dynamics can be problematic if they are in the same time scales. A related approach to compute the required spectral densities is the reorientational eigenmode dynamics developed by Brüschweiler and co-workers. ${ }^{[25]} \mathrm{Fi}-$ nally, for systems such as peptides which are small enough to sufficiently sample all, internal and rotational, motional processes in an MD simulation, all interproton vector time correlation functions and spectral densities can be computed, and the relaxation-matrix equation can be solved. A procedure to carry out this back-calculation of NOESY and ROESY spectra from MD simulations of peptides has been implemented and tested before. ${ }^{[26,27]}$

Here, we present a structural analysis of an $\mathrm{N}$-acetylated and $\mathrm{C}$-amidated $\beta$-dipeptide with residues homologuous to valine and phenylalanine (Val-Phe, see Figure 1), which serves as a small test system to investigate the influence of the substitution pattern on the formation of hydrogen bonds as described above. The theoretically possible hydrogen bonds and the nomenclature used are also shown in Figure 1. The specific substitution pattern (the N-terminal residue $\alpha$-substituted and the $\mathrm{C}$-terminal residue $\beta$-substituted) and the side-chain types were chosen since this dipeptide is a fragment of a longer peptide for which helix formation had been observed. ${ }^{[3]}$ Predictions-based on chemical intuition, experience from larger oligomers ${ }^{[3]}$ and preliminary ab initio modelling — on the conformations preferentially adopted by this dimer, are different for the two types of diastereomers: the $S S$ (or $R R$ ) diastereomer is predicted to form a 10-membered hydrogen bond, whereas no preferential hydrogen bonding is predicted for the $S R$ (or $R S$ ) diastereomer. The present study focuses on two major topics, the conformational analysis of the dipeptides with the help of MD simulations, and the NMR analysis, where we compare simulated and experimental ${ }^{1} \mathrm{H}$ ROESY spectra and ${ }^{3} J$ coupling constants. For the conformational analysis the influence of the backbone stereocenters and the side chains on the hydrogen bonding and the backbone torsional angles is investigated, and the simulated structures are grouped into so-called clusters of structures each representing a conformation. By performing not only MD simulations of the 
A)

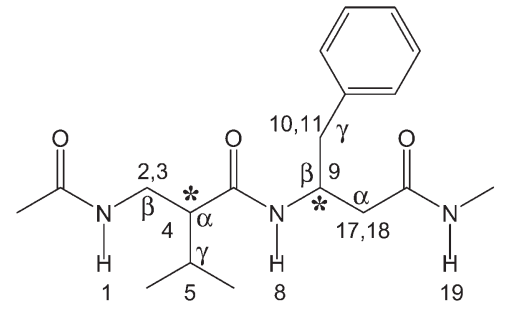

B)

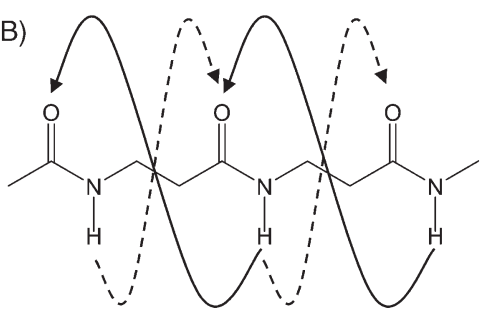

$\begin{array}{llll}\mathrm{HB}_{6 \mathrm{a}} & \mathrm{HB}_{8 \mathrm{a}} & \mathrm{HB}_{6 \mathrm{~b}} & \mathrm{HB}_{8 \mathrm{~b}}\end{array}$

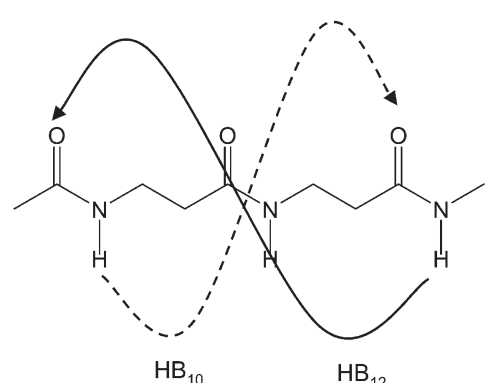

$\mathrm{HB}_{10}$

$\mathrm{HB}_{12}$

Figure 1. A) chemical formula of the investigated $\beta$-dipeptide Val-Phe, * indicates the two stereocenters, the numbers refer to more closely investigated protons, the Greek letters to the carbon atoms. B) Theoretically possible hydrogen bonds (HB) and the nomenclature used.

experimentally accessible $S R$ and $S S$ isomers of Val-Phe at $298 \mathrm{~K}$, but also simulations of Val-Phe at $340 \mathrm{~K}$ and of the corresponding $\beta$-dipeptides with residues homologuous to alanine (di-Ala, both $S R$ and $S S$ ) and glycine at $298 \mathrm{~K}$, that is, the corresponding $\beta$-dipeptides with methyl groups as side chains and the one without any side chain, one obtains a more precise picture of hydrogen-bond and secondarystructure formation.

In the NMR analysis, the influence of structural and dynamical properties on NMR (particularly ROESY) spectra is tested. This offers the possibility to assess the sensitivity of the spectra to structural changes and consequently the significance of the data obtained from these spectra for structure prediction of such small systems. Three methods to back-calculate the spectra from the MD trajectories with varying levels of accuracy are used and denoted as follows:

- flexible: The spectra are computed by determining time correlation functions and spectral densities for all proton pairs, inserting the relaxation rates into the relaxation matrix and solving the relaxation-matrix equation. This method accounts for all aspects of internal dynamics (fluctuating distances, individual correlation times, "orientational" effects), correlation with overall dynamics and spin diffusion.

- rigid: No time correlation functions are computed, but the $\left\langle r^{-6}\right\rangle$-averaged distances of all proton pairs are inserted into an analytical function for the spectral density for an isotropically tumbling rigid molecule. These spectral densities are used to solve the relaxation-matrix equation. This method accounts only for fluctuating distances and spin diffusion.

- naive: Here, the spectral intensities are naively assumed to be proportional to the $\left\langle r^{-6}\right\rangle$-averaged distances of the corresponding interproton vectors, which corresponds to the conventional method to compare distance averages from the simulation with experimentally derived distances (initial-rate approximation).

Comparison of the spectra obtained with these approaches allows to test the influence of the above-mentioned aspects of internal dynamics on the spectra and to evaluate the various methods to analyze NMR relaxation data, particularly with regard to small flexible molecules.

We address a number of questions. Does one observe the (experimentally) predicted conformational differences between the $S R$ and the $S S$ isomers of Val-Phe in the MD simulations at $298 \mathrm{~K}$ ? Do the MD simulations reproduce the experimental data, particularly the ROESY spectra? Does the flexible approach reproduce the experimental spectra better than the rigid and naive ones? Upon comparing the simulated spectra of the $S R$ and $S S$ isomers, does one observe significant differences, that is, are conformational differences reflected in the NMR spectra? Upon modifying the simulation conditions (temperature, side chains): do the MD simulations sample different structures? Which effects does this have on the ROESY spectrum?

\section{Results and Discussion}

$35 \mathrm{~ns}$ MD simulations at constant temperature $(298 \mathrm{~K}$ or $340 \mathrm{~K}$ ) and constant pressure (1 atm) of the following molecules in methanol were carried out using the GROMOS package $^{[28,29]}$ and the GROMOS 43A1 force field ${ }^{[29]}$ : the $S R$ and $S S$ diastereomers of Val-Phe as shown in Figure 1 at 298 and at $340 \mathrm{~K}$ (denoted as Val-Phe ${ }_{S R, 298}$, Val-Phe ${ }_{S S, 298}$, Val$\mathrm{Phe}_{S R, 340}$ and Val-Phe ${ }_{S S, 340}$ ), the corresponding $S R$ and $S S$ diastereomers of Ala-Ala at $298 \mathrm{~K}\left(\right.$ di-Ala $_{S R}$ and di-Ala $\left.S S\right)$ and of Gly-Gly at $298 \mathrm{~K}$ (di-Gly). The resulting trajectories were analyzed using the cluster algorithm described by Daura et al. ${ }^{[30]}$ (see also Computational methods section), the numbering of the clusters of structures is according to their relative population: cluster 1 is most populated, etc. The present section is organized as follows: first, we describe the MD simulations of the two diastereomers of Val-Phe at $298 \mathrm{~K}$, analyze the resulting conformations particularly with respect to the predicted structural differences and compare simulated and experimental data, namely ${ }^{1} \mathrm{H}$ ROESY spectra and ${ }^{3} J$ coupling constants. In the second part, we investigate more closely possible effects of the method to compute the ROESY spectra. Third, the influence of the temperature on 
the simulated ensembles will be studied, and fourth, the influence of the side chains is considered.

Val-Phe $_{S R, 298}$ and Val-Phe ${ }_{S S, 298}$ - Influence of the stereocenters: Figure 2 presents the central member structures of the most populated clusters of simulations Val-Phe $\operatorname{VR}_{S R, 298}(\mathrm{~A})$ and Val-Phe $_{S S, 298}(\mathrm{C})$. The $S R$ isomer shows an open, S-shaped

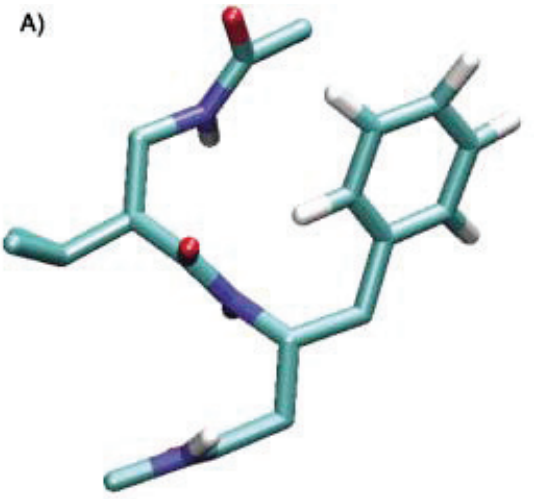

$16 \%\left(\mathrm{HB}_{10} ; 0 \%\right)$

C)

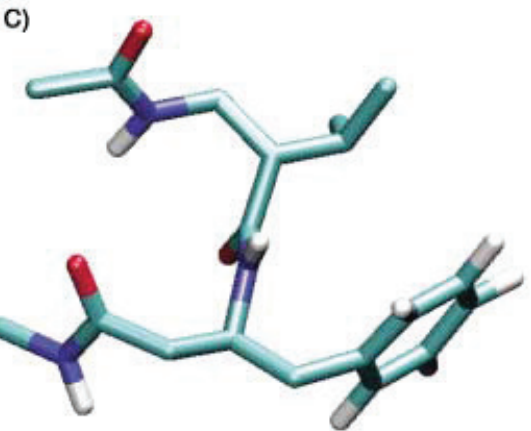

$58 \%\left(\mathrm{HB}_{10}: 60 \%\right)$

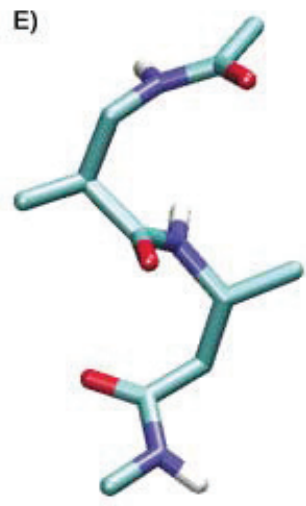

$15 \%\left(\mathrm{HB}_{10}: 0 \%\right)$
B)

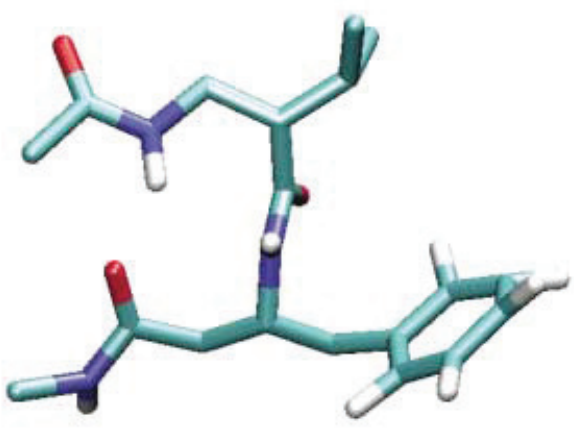

$17 \%\left(\mathrm{HB}_{10}: 53 \%\right)$

D)
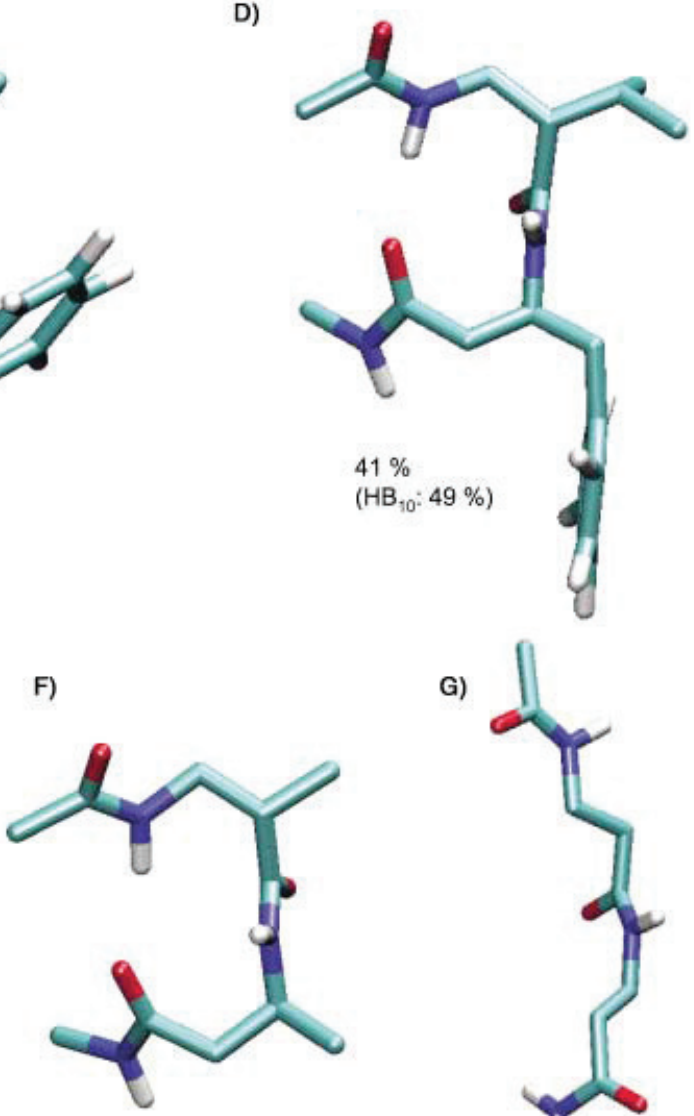

$50 \%\left(\mathrm{HB}_{10}: 54 \%\right)$
G)

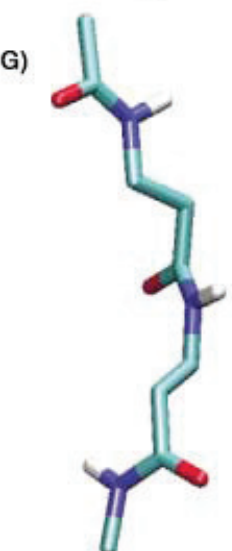

$4 \%\left(\mathrm{HB}_{10}: 0 \%\right)$
Figure 2. Central member structures of the most populated clusters in the MD simulations, the corresponding relative populations of these clusters and the 10 -membered ring hydrogen bond $\left(\mathrm{HB}_{10}\right.$, see Figure 1$)$ populations therein: A) Val-Phe $e_{S R, 298}$; B) Val-Phe ${ }_{S R, 340}$; C) Val-Phe $\mathrm{V}_{S, 298}$; D) Val-Phe $\mathrm{V}_{S S, 340}$; E) di-Ala $\mathrm{Al}_{S R, 298}$; F) di-Ala $\mathrm{S}_{S S, 298}$; G) di-Gly 298 . conformation, whereas the SS isomer exhibits a closed struc$60 \%$ of all structures in cluster 1 (see Figure 2C) and in of all structures in the complete simulation ValPhe $_{S S, 298}$ (see Table 1). In contrast, $\mathrm{HB}_{10}$ is not at all found in simulation Val-Phe ${ }_{S R, 298}$. Table 1 summarizes the hydrogenbond populations in all MD simulations performed (of those hydrogen bonds which appear for more than $1 \%$ in any of the simulations). Figure 3 monitors the formation of hydrogen bonds throughout the simulations and the atom-positional root-mean-square distance (RMSD) of the structures in the simulations from the central member structure of the corresponding most populated clusters. Simulation Val-Phe ${ }_{S R, 298}$ does not show any significant hydrogen bonding at all. Only $\mathrm{HB}_{8 \mathrm{~b}}$ is formed in $2 \%$ of the structures (Table 1), but it cannot be attributed to a specific conformation or cluster or to a specific period in the simulation, it appears transiently in many clusters at many time points in the simulation (see Figure 3A). Simulation ValPhe $_{S S, 298}$ (Figure 3C) shows-in addition to the dominant 10 membered ring structure formed by $\mathrm{HB}_{10}$ and to $\mathrm{HB}_{8 \mathrm{~b}}$, which is also here formed only transiently-a 12-membered ring structure formed by $\mathrm{HB}_{12}$. $\mathrm{HB}_{12}$ is present in $2 \%$ of all structures (Table 1) and predominantly in specific clusters (e.g. for $28 \%$ in cluster 3 and for $42 \%$ in cluster 11 ), which indicates that this hydrogen bond defines a specific conformation. Looking at the populations of the predominant clusters of Val-Phe ${ }_{S R, 298}(16 \%)$ and Val-Phe $_{S S, 298}(58 \%)$ (Figure 2A and $\mathrm{C}$ ), one can see that the most populated cluster is less representative of the complete simulation in case of Val$\mathrm{Phe}_{S R, 298}$ than in case of Val$\mathrm{Phe}_{S S, 298}$. This shows, together with the different hydrogen bonding patterns, that the $S R$ isomer has less well-defined 
Table 1. Hydrogen-bond populations (in \% reported if population $>1 \%$ ) in seven simulations of different $\beta$ dipeptides in methanol. The hydrogen bonds are defined in Figure 1 and the hydrogen-bond criterion is a maximum distance of $0.25 \mathrm{~nm}$ between hydrogen and acceptor atom and a minimum angle of $135^{\circ}$ between donor, hydrogen and acceptor. The different stereoisomers at the central $\alpha$ - and $\beta$-carbons are denoted by $S$ and $R$. The indices denote temperatures.

\begin{tabular}{|c|c|c|c|c|c|c|c|}
\hline & & \multicolumn{3}{|c|}{$S S$} & \multirow[b]{2}{*}{ di-Gly $_{298}$} \\
\hline & Val-Phe $_{298}$ & Val-Phe ${ }_{340}$ & di-Ala 298 & Val-Phe ${ }_{298}$ & Val-Phe ${ }_{340}$ & di-Ala 298 & \\
\hline$\overline{\mathrm{HB}_{8 \mathrm{a}}}$ & - & 1.2 & 1.6 & - & - & - & - \\
\hline $\mathrm{HB}_{8 \mathrm{~b}}$ & 1.9 & 2.5 & 2.1 & 1.1 & 2.1 & 1.5 & - \\
\hline $\mathrm{HB}_{10}$ & - & 13.0 & 4.4 & 38.7 & 25.4 & 29.6 & 2.8 \\
\hline $\mathrm{HB}_{12}$ & - & 3.6 & - & 2.1 & 3.6 & 2.1 & 2.0 \\
\hline
\end{tabular}

terms of correlation. This agreement is confirmed by the corresponding correlation coefficients (96 to $94 \%$ ) given in Table 2 (upper part, first line) for both mixing times.

Table 3 shows experimental and simulated ${ }^{3} J_{\mathrm{H}^{N} \mathrm{H}^{\mathrm{C}}}$ and ${ }^{3} J_{\mathrm{H}^{\mathrm{C}} \mathrm{H}^{\mathrm{C}}}$ coupling constants, the ${ }^{3} J_{\mathrm{H}^{\mathrm{C}} \mathrm{H}^{\mathrm{C}}}$ values were computed from the simulations using one set of Karplus coefficients, ${ }^{[31]}$ the structural preferences and is more flexible. On the other hand, for both isomers the RMSD from cluster 1 remains low for most of the time (Figure 3A and C), which means that most of structures of the $S R$ isomer resemble the open, $\mathrm{S}$-shaped conformation, whereas the $S S$ isomer shows some sort of ring structure for most of the time. Summarizing, it can be said that these results confirm the predictions about the hydrogen-bond formation of the two stereoisomers at room temperature. The dominance of the 10- over the 12membered ring is in good agreement with observations made with longer $\beta$-peptides, where it was found that conformationally restricted amide bonds with two neighbouring substituents induce the formation of 10-membered hydrogenbonded rings, whereas amide bonds without adjacent substituents favour 12-membered rings. ${ }^{[3]}$

In order to compare the results obtained from the MD simulations with experimental data, the ${ }^{1} \mathrm{H}$ ROESY spectra at two mixing times $t_{\mathrm{m}}=100$ and $150 \mathrm{~ms}$ and a set of $10^{3} \mathrm{~J}$ coupling constants were analyzed. Figure 4 shows that the agreement of simulated ROESY intensities computed from simulations Val-Phe VR,298 (left) and Val-Phe ${ }_{S S, 298}$ (right) using the flexible (full circles, solid line) and the rigid (empty squares, dashed line) approach and experimental intensities recorded at $298 \mathrm{~K}$ after a mixing time of $100 \mathrm{~ms}$ is very good. Since the absolute intensities in the experimental spectra depend on the exact circumstances of the measurement and on the exact concentration in the probe, intensities are not compared in absolute numbers but only in
Table 2. Correlation of experimental and simulated intensity for the Val-Phe $\beta$-dipeptides in methanol. ${ }^{\text {[a }}$

\begin{tabular}{|c|c|c|c|c|c|}
\hline & & \multicolumn{4}{|c|}{ Experiment } \\
\hline & & \multicolumn{2}{|c|}{$S R$} & \multicolumn{2}{|c|}{$S S$} \\
\hline & & $t_{\mathrm{m}}=100 \mathrm{~ms}$ & $t_{\mathrm{m}}=150 \mathrm{~ms}$ & $t_{\mathrm{m}}=100 \mathrm{~ms}$ & $t_{\mathrm{m}}=150 \mathrm{~ms}$ \\
\hline \multirow[t]{6}{*}{ simulation } & flexible & 0.96 & 0.95 & 0.94 & 0.94 \\
\hline & rigid & 0.88 & 0.86 & 0.85 & 0.84 \\
\hline & naive & 0.87 & 0.85 & 0.85 & 0.83 \\
\hline & & \multicolumn{4}{|c|}{ Simulation (flexible) } \\
\hline & & \multicolumn{2}{|c|}{$S R$} & \multicolumn{2}{|c|}{$S S$} \\
\hline & & $298 \mathrm{~K}$ & $340 \mathrm{~K}$ & $298 \mathrm{~K}$ & $340 \mathrm{~K}$ \\
\hline \multirow[t]{2}{*}{ experiment } & $S R$ & 0.96 & 0.75 & 0.91 & 0.87 \\
\hline & $S S$ & 0.90 & 0.66 & 0.94 & 0.92 \\
\hline
\end{tabular}

[a] Different stereoisomers $(R, S)$ at Phe, different simulation temperatures $(298,340 \mathrm{~K})$, different mixing times $\left(t_{\mathrm{m}}\right)$ and different motional models (flexible, rigid, naive) are considered. Upper part of table: "correct" comparison of simulation with corresponding experiment (Graphs see Figure 4); lower part: cross-comparison with $t_{\mathrm{m}}=100 \mathrm{~ms}$ (see Figure 6).

Table 3. Comparison of simulated and experimental ${ }^{3} J$ coupling constants $[\mathrm{Hz}]$ for the Val-Phe $\beta$-dipeptides in methanol. Different stereoisomers $(R, S)$ at Phe and different simulation temperatures are considered. ${ }^{\text {[a] }}$

\begin{tabular}{|c|c|c|c|c|c|c|c|}
\hline \multirow[b]{2}{*}{ Protons } & \multirow[b]{2}{*}{ Isomer } & \multicolumn{4}{|c|}{ Simulated (298 K) } & \multirow{2}{*}{$\begin{array}{l}(340 \mathrm{~K}) \\
\text { (a) }\end{array}$} & \multirow[t]{2}{*}{ Exptl (298 K) } \\
\hline & & (a) & (b) & (c) & (d) & & \\
\hline \multirow{2}{*}{${ }^{3} J_{\mathrm{H}^{\mathrm{N}} \mathrm{H}^{\mathrm{\beta}}, 1}(\mathrm{Val})$} & $S R$ & 5.4 & 5.4 & 5.5 & 4.9 & 5.7 & 6.1 \\
\hline & $S S$ & 6.5 & 6.9 & 6.7 & 6.1 & 6.1 & 7.2 \\
\hline \multirow[t]{2}{*}{${ }^{3} J_{\mathrm{H}^{\mathrm{N}} \mathrm{H}^{\beta, 2}}(\mathrm{Val})$} & $S R$ & 6.1 & 6.4 & 6.3 & 5.8 & 5.8 & 5.8 \\
\hline & $S S$ & 5.4 & 5.5 & 5.5 & 5.0 & 5.7 & 4.8 \\
\hline \multirow{2}{*}{${ }^{3} J_{\mathrm{H}^{\beta, 1} \mathrm{H}^{\alpha}}(\mathrm{Val})$} & $S R$ & 8.2 & & & & 8.8 & 9.2 \\
\hline & $S S$ & 11.0 & & & & 9.8 & 9.9 \\
\hline \multirow[t]{2}{*}{${ }^{3} J_{\mathrm{H}^{\beta}, 2 \mathrm{H}^{\alpha}}(\mathrm{Val})$} & $S R$ & 4.5 & & & & 4.2 & 4.5 \\
\hline & $S S$ & 3.8 & & & & 4.3 & 4.2 \\
\hline \multirow[t]{2}{*}{${ }^{3} J_{\mathrm{H}^{\alpha} \mathrm{H}^{\gamma}}(\mathrm{Val})$} & $S R$ & 7.2 & & & & 6.8 & 8.5 \\
\hline & $S S$ & 6.2 & & & & 6.1 & 8.4 \\
\hline \multirow[t]{2}{*}{${ }^{3} J_{\mathrm{H}^{\mathrm{N}} \mathrm{H}^{\beta}}(\mathrm{Phe})$} & $S R$ & 7.0 & 7.4 & 7.2 & 6.6 & 6.1 & 8.4 \\
\hline & $S S$ & 7.1 & 7.5 & 7.3 & 6.7 & 6.2 & 8.4 \\
\hline \multirow[t]{2}{*}{${ }^{3} J_{\mathrm{H}^{\beta} \mathrm{H}^{\alpha, 1}}(\mathrm{Phe})$} & $S R$ & 4.0 & & & & 4.1 & 5.8 \\
\hline & $S S$ & 3.5 & & & & 4.2 & 5.3 \\
\hline \multirow[t]{2}{*}{${ }^{3} J_{\mathrm{H}^{\beta} \mathrm{H}^{\alpha, 2}}$ (Phe) } & $S R$ & 10.8 & & & & 10.6 & 8.2 \\
\hline & $S S$ & 11.3 & & & & 10.3 & 8.3 \\
\hline${ }^{3} J_{\mathrm{H}^{\beta} \mathrm{H}^{\gamma, 1}}(\mathrm{Phe})$ & $S S$ & 3.9 & & & & 4.2 & 5.0 \\
\hline${ }^{3} J_{\mathrm{H}^{\beta} \mathrm{H}^{\gamma / 2}}(\mathrm{Phe})$ & $S S$ & 11.0 & & & & 10.6 & 9.2 \\
\hline
\end{tabular}

[a] Parameters of the Karplus Equation (1) were taken according to a) refs. [31,32], b) ref. [33], c) ref. [34], and d) ref. [35], for details see Computational Methods and Table 4. 
A)

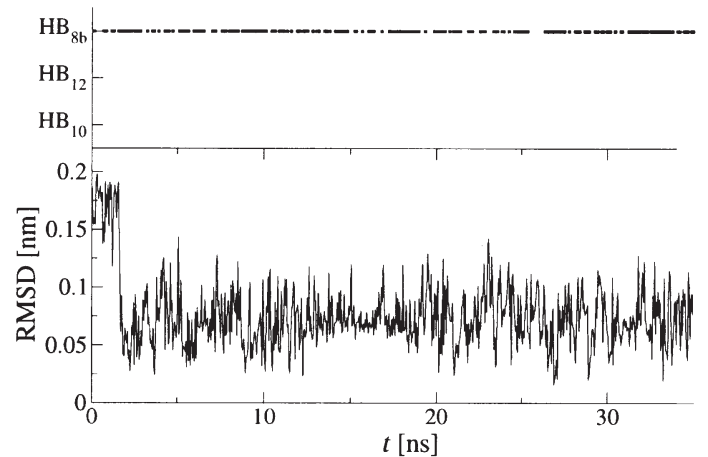

C)

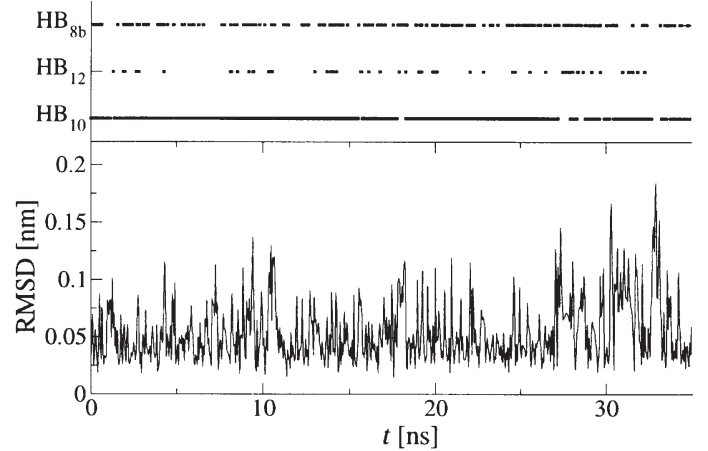

E)

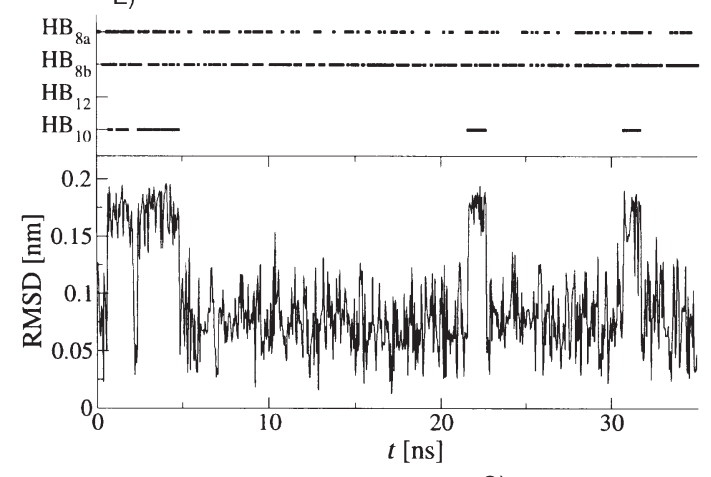

G)

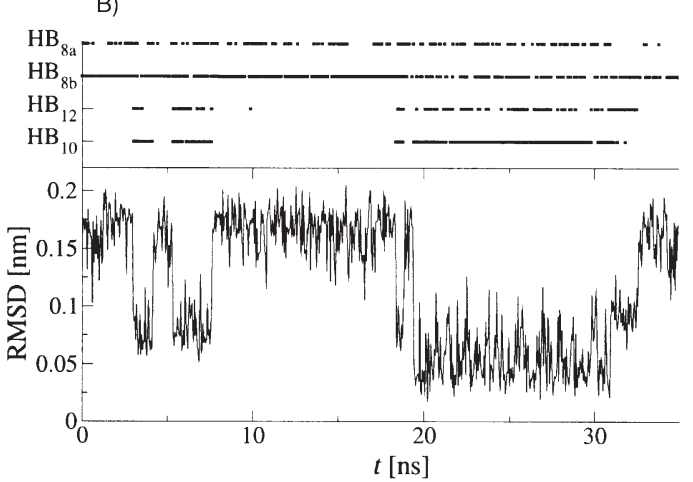

D)

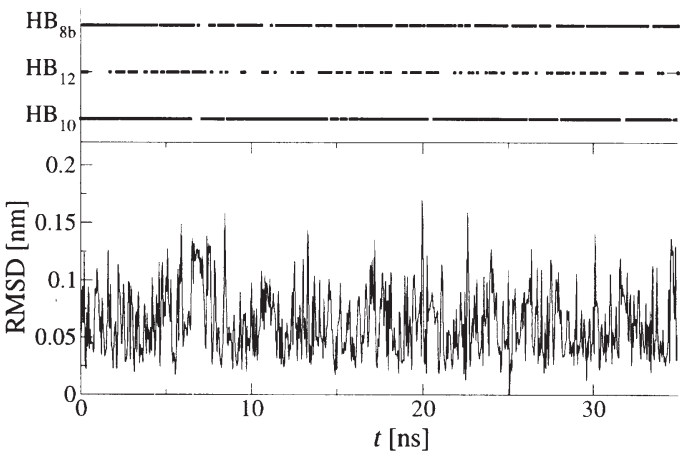

F)

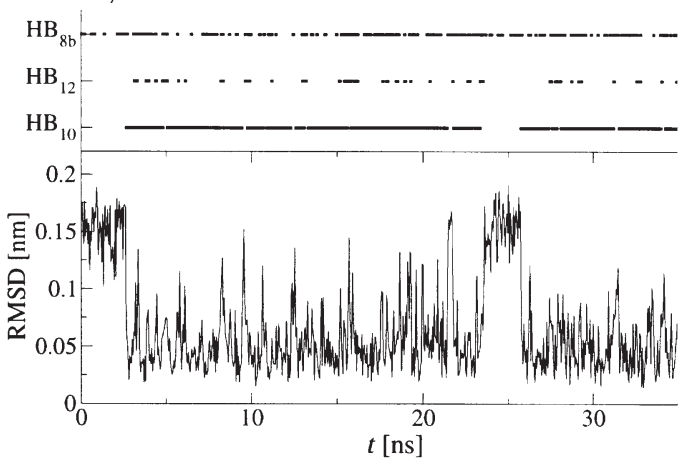

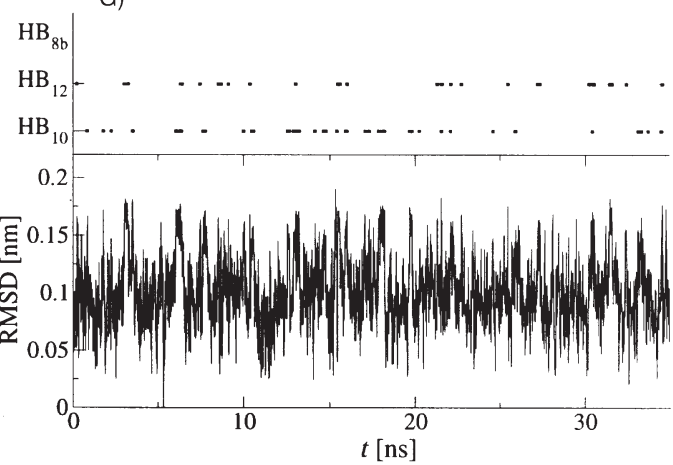

Figure 3. Backbone atom-positional root-mean-square distance (RMSD) of structures in the simulations from the corresponding central member structure of cluster 1 (see Figure 2) and occurrence of some hydrogen bonds as a function of time: A) Val-Phe $S_{S R, 298}$; B) Val-Phe ${ }_{S R, 340}$; C) Val-Phe $S_{S S, 298}$; D) Val$\left.\left.\mathrm{Phe}_{S S 340} ; \mathrm{E}\right) \mathrm{di}-\mathrm{Ala}_{S R 298} ; \mathrm{F}\right)$ di-Ala $\left.{ }_{S S 298} ; \mathrm{G}\right)$ di-Gly $_{298}$. For a definition of the hydrogen bonds, see Figure 1. Due to low plotting resolution a hydrogen bond may be on average less present than implicated by the figure.

correctly predict which of the two isomers yields the larger ${ }^{3} J$ value. In the next section the back-calculation of the ROESY spectra is more closely discussed, especially with respect to the applied method, the sensitivity of the intensities to dynamics and structural changes, and as a conse- quence the relevance of the good agreement between simulation and experiment for structure prediction.

Val-Phe $_{S R, 298}$ and Val-Phe ${ }_{S S, 298}$-Simulated ROESY spectra: Table 2 (upper part) presents the correlation coefficients be- 

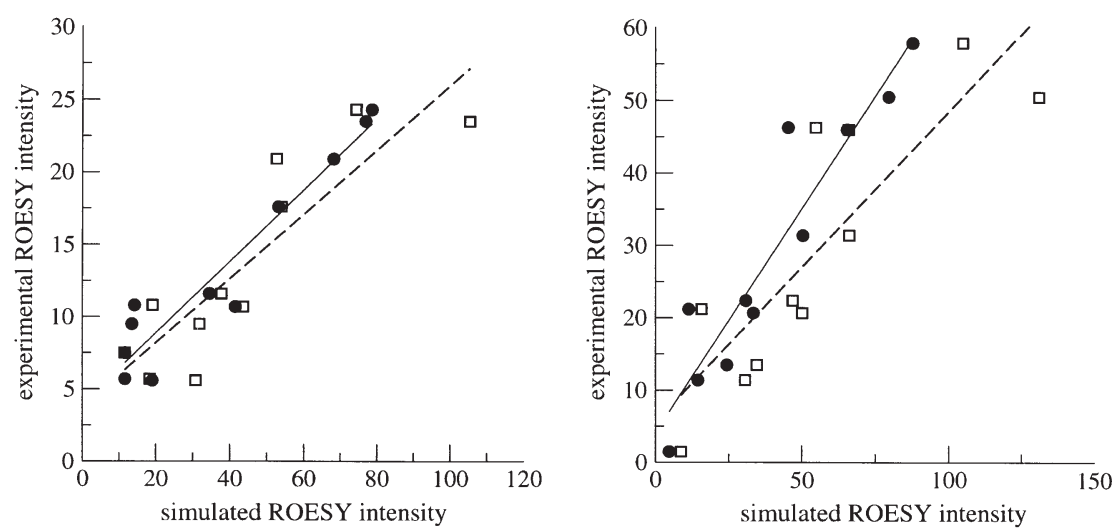

Figure 4. Comparison of experimental and simulated (at $298 \mathrm{~K}$ ) ROESY intensities at a mixing time of $100 \mathrm{~ms}$ Left: Val-Phe ${ }_{S R}$; right: Val-Phe ${ }_{S S}$; $\bullet$ flexible approach; $\square$ rigid approach; —_ linear fit for flexible; -----: linear fit for rigid. The intensities cannot be compared in absolute numbers, only in terms of correlation. Correlation coefficients can be found in Table 2 .

tween experimental intensities of the spectra of the two isomers and theoretical intensities obtained from the MD simulations using the three above-mentioned approaches, that is, flexible, rigid and naive. The agreement of the flexible spectra with the experiments (94 to $96 \%$ correlation) is significantly better than that for the rigid ( 84 to $88 \%$ ) and naive ( 83 to $87 \%$ ) ones, for example, the difference in correlation between the flexible and the rigid approach lies between 8 and $10 \%$, which means that the inclusion of all aspects of internal dynamics is relevant for the correct prediction of NMR relaxation parameters of such small molecules. The correlation coefficients of the rigid and naive approaches, however, are almost identical, with the maximal difference between the two approaches being $1 \%$. This means that for this system and these mixing times spin-diffusion effects are negligible. In order to assess the influence of the structural differences between the two isomers on the spectral intensities, the simulated spectra of the two stereoisomers computed from the various simulations are compared to each other. Figure 5A shows the simulated intensities computed from Val-Phe ${ }_{S S, 298}$ versus those from Val-
$\mathrm{Phe}_{S R, 298}$ with the flexible approach, Figure 5B those using the rigid approach. Proton pairs for which the intensities differ significantly between the two isomers are indicated in the figure. The largest deviations are found within the first residue (Val). The relative intensity differences between the two isomers are a little larger when calculated with the flexible than with the rigid method, which shows that they are at least partly due to differences in internal dynamics. In spite of the large structural differences between the isomers, the differences in the spectra are limited to a very small set of peaks. In Figure 5 it is also indicated which of these peaks would be observable in the true spectrum (squares), that is, superposition is taken into account. Obviously the anyway meagre differences between the isomers are even partly compensated by superposition. To estimate the significance of these differences with respect to the experimental data, Figures 6A and B show a cross comparison of the intensities computed from the simulations Val-

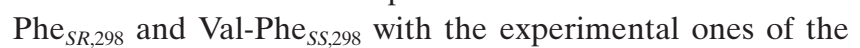
$S S$ and the $S R$ isomers. The corresponding correlation coefficients are given in Table 2 (lower part). In the Figure, those peaks which should stand out (from what we know from Figure 5A) are highlighted. The conclusion of this cross comparison is, that it is indeed very difficult to detect the structural differences between the isomers in the experimental spectra.

Val-Phe $_{S R, 340}$ and Val-Phe ${ }_{S s, 340}$-Influence of temperature: Increasing the temperature has very different effects on the two diastereomers. The $S S$ isomer does not show strong differences between the two temperatures. The 10-membered
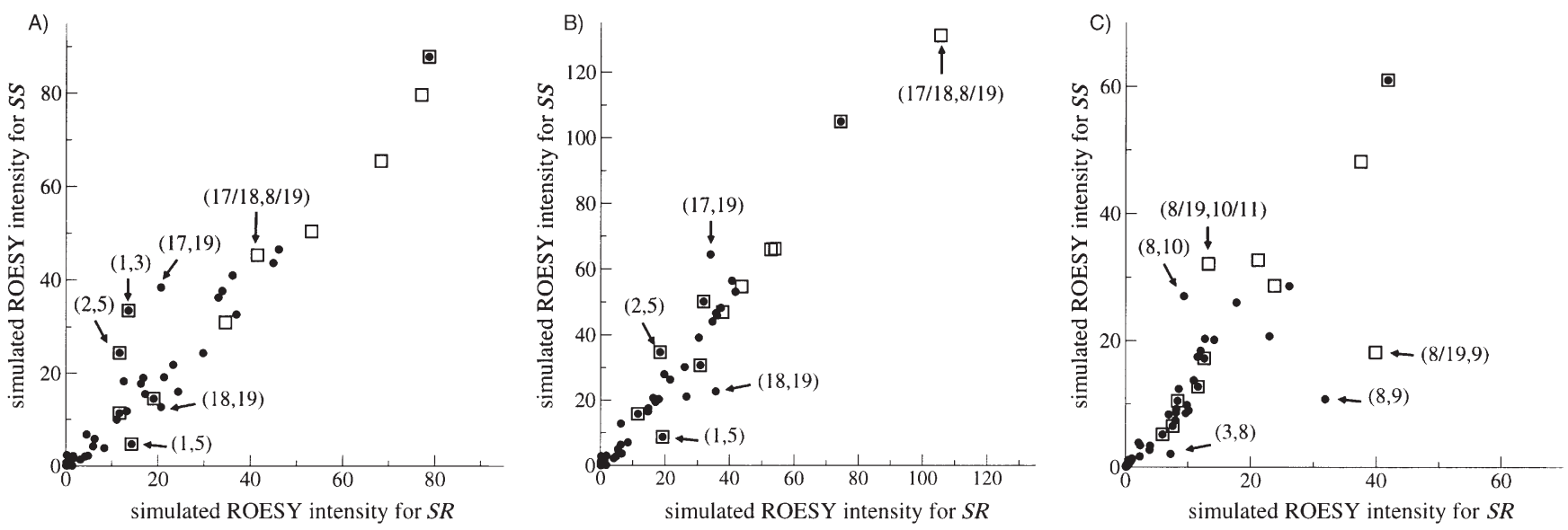

Figure 5. Comparison of simulated ROESY intensities of the $S S$ and the $S R$ isomers $\left(t_{\mathrm{m}}=100 \mathrm{~ms}\right)$. A) Val-Phe $S S, 298$ vs Val-Phe $\mathrm{VR}_{2,298}($ flexible); B: Val$\mathrm{Phe}_{S S, 298}$ vs Val-Phe $\mathrm{S}_{S R, 298}\left(\right.$ rigid); C) Val-Phe ${ }_{S S, 340}$ vs Val-Phe $_{S R, 340}$ (flexible). • simulated intensities; $\square$ simulated intensities translated to observable peaks (accounting for superposition of peaks in the experiment). Arrows indicate special proton pairs (for numbering see Figure 1). 
ring is still the predominant conformation (see Figure 2D), hydrogen-bond populations are very similar (see Table 1), $\mathrm{HB}_{10}$ is formed a little less, $\mathrm{HB}_{12}$ slightly more often than at lower temperature. For the $S R$ isomer, however, the situation changes dramatically upon increasing the temperature from 298 to $340 \mathrm{~K}$. The predominant structure is now a $10-$ membered ring (see Figure $2 \mathrm{~B}$ ). $\mathrm{HB}_{10}$ is found in $13 \%$ of all structures, and $\mathrm{HB}_{12}$ is formed as well $(\approx 4 \%)$. This means that in terms of the ring structures found, the simulations of the two isomers are similar at higher temperature. Nevertheless, the $S S$ isomer is still structurally more stable than the $S R$ isomer, as can be seen in Figure 3, where the $S R$ isomer unfolds many times to the extended conformation (Figure $3 \mathrm{~B}$ ), whereas the RMSD of the $S S$ isomer remains low with respect to the first cluster throughout the whole simulation (Figure 3D). The first cluster has a relative population of $41 \%$ in case of Val-Phe ${ }_{S S, 340}$ but only of $17 \%$ in case of Val-Phe ${ }_{S R, 340}$ (Figure 2), and $\mathrm{HB}_{10}$ is observed for 25 and $13 \%$, respectively (Table 1 ). In order to study the impact of the structural changes that occur when increasing the temperature, the ${ }^{3} J$ coupling constants and the ROESY spectra were calculated from simulations Val-Phe ${ }_{S R, 340}$ and Val$\mathrm{Phe}_{S S, 340}$ as well. No larger effects on the ${ }^{3} J$ coupling constants can be observed, the overall agreement with the experimental ${ }^{3} J$ values neither improved nor deteriorated compared with the simulations at $298 \mathrm{~K}$. The cross comparison between the simulated spectra for the two stereoisomers at $340 \mathrm{~K}$ and the experimental spectra is presented in Figures $6 \mathrm{C}$ and $\mathrm{D}$. The corresponding correlation coefficients are given in the lower part of Table 2. The spectrum computed from Val-Phe ${ }_{S S, 340}$ is in comparatively good agreement with the experimental spectrum of the $S S$ isomer (recorded at room temperature), but as well in reasonable agreement with the experimental spectrum of the $S R$ isomer, whereas the spectrum computed from Val$\mathrm{Phe}_{S R, 340}$ much more severely deviates from both experimental spectra. This indicates that in simulation Val-Phe ${ }_{S R, 340}$ a process occurs which does not correctly reproduce the experimental equilibrium at $298 \mathrm{~K}$ of either diastereomer. In addition to the comparison with experimental intensities, the simulated spectra computed from Valnumbering see Figure 1).
Phe $_{S S, 340}$ (with the flexible method) are compared to those computed from Val-Phe ${ }_{S R, 340}$ in Figure 5C. Compared with panel A, where the same is done for the simulations at $298 \mathrm{~K}$, one sees that the deviations between $S S$ and $S R$ isomer occur for completely different proton pairs, the differences are now located around the central peptide bond, whereas at lower temperature they are found within the first residue. In order to understand this observation in terms of molecular structure and dynamics, the values of the backbone dihedral angles (including the first side chain dihedral angle) were investigated. This study of the torsional angle profiles also helps to interpret the observed, at first sight counterintuitive enhancement of hydrogen bonding (structure formation) in the $S R$ isomer upon temperature increase, for which one could give two possible explanations, i) the sampling of dihedral angle transitions is not sufficient at lower temperature, an explanation which would be supported by major changes in the torsional angle profiles of the simulations between the two temperatures, or ii) an entropic effect where the balance between intramolecular hydrogen bonding and intermolecular hydrogen bond forma-
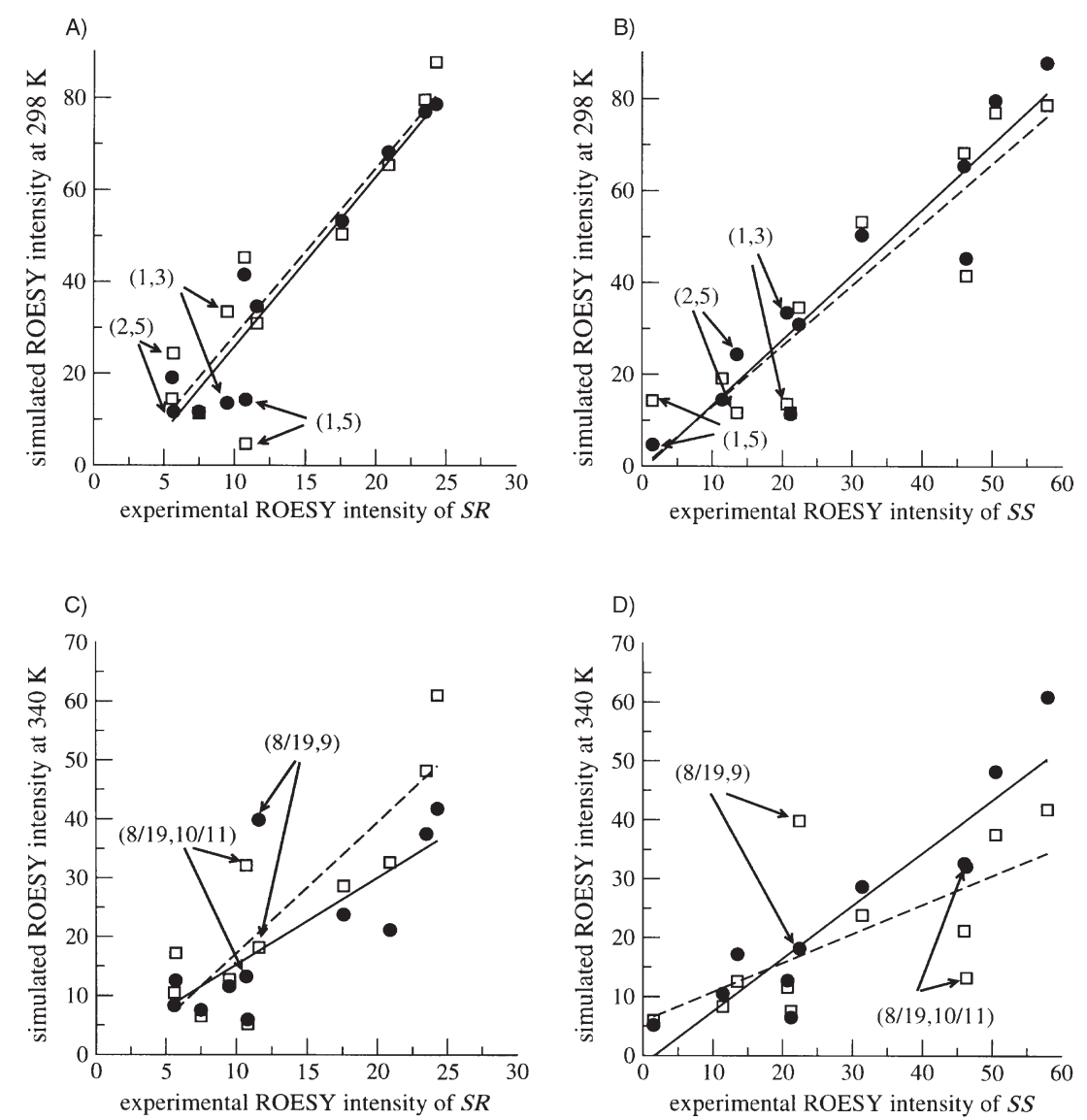

Figure 6. Cross comparison of simulated and experimental ROESY intensities at $t_{\mathrm{m}}=100 \mathrm{~ms}$. A: Simulated intensities of Val-Phe ${ }_{S R, 298}$ (full circles and solid line) and Val-Phe ${ }_{S S, 298}$ (empty squares and dashed line) vs experimental intensities of the $S R$ isomer $\left(298 \mathrm{~K}\right.$ ). B) Simulated intensities of Val-Phe ${ }_{S S, 298}$ (full circles and solid line) and Val-Phe ${ }_{S R, 298}$ (empty squares and dashed line) vs experimental intensities of the $S S$ isomer (298 K). C) Simulated intensities of Val-Phe $S_{S R, 340}$ (full circles and solid line) and Val-Phe ${ }_{S S, 340}$ (empty squares and dashed line) vs experimental intensities of the $S R$ isomer $(298 \mathrm{~K})$. D) Simulated intensities of Val-Phe ${ }_{S S, 340}$ (full circles and solid line) and Val-Phe ${ }_{S R, 340}$ (empty squares and dashed line) vs experimental intensities of the $S S$ isomer $(298 \mathrm{~K})$. Correlation coefficients can be found in Table 2. Arrows indicate special proton pairs (for 
tion with solvent molecules changes with temperature. Figure 7A shows the torsional profiles in simulations ValPhe $_{S R, 298}$ (grey) and Val-Phe ${ }_{S S, 298}$ (striped). For the second residue (right panels), the torsional profiles of the $S R$ and $S S$ isomers are mirror images (around $180^{\circ}$ ) due to the inversion of the stereocenter in this residue. The largest difference between the two simulations appears in the dihedral $\left(b_{1}\right)$ around the bond between the $\mathrm{C}_{\beta}$ and $\mathrm{C}_{\alpha}$ atom (and slightly in the dihedral $\left(c_{1}\right)$ around the bond between the $\mathrm{C}_{\alpha}$ and the carbonyl $\mathrm{C}$ atom) in the first residue, where the $S R$ isomer samples an additional minimum. This is probably responsible for the fact, that the largest differences between the spectra computed from Val-Phe ${ }_{S S, 298}$ and Val-Phe ${ }_{S R, 298}$ (Figure 5A) are found within the first residue. For several other dihedrals, the torsional angle profiles of the $S R$ isomer (grey) are more symmetric than those of the $S S$ isomer (striped). All these differences reflect that the $S S$ isomer adopts a comparatively rigid ring conformation, whereas the $S R$ isomer is rather extended and flexible. Figure 7B presents the same profiles for the corresponding simulations at $340 \mathrm{~K}$. Two major observations can be made, i) the $S S$ isomer now also samples the third conformation (at $300^{\circ}$ ) of the torsional angle $\left(b_{1}\right)$ around the $C_{\beta}-C_{\alpha}$ bond in residue 1 , which explains why the differences for proton pairs in the first residue in the spectra vanishes going from Figure $5 \mathrm{~A}$ to $\mathrm{C}$, and ii) simulation Val-Phe ${ }_{S R, 340}$ starts sampling new conformations $\left(\right.$ at $\left.300^{\circ}\right)$ of the torsional angle $\left(\mathrm{a}_{2}\right)$ around the $\mathrm{N}-\mathrm{C}_{\beta}$ bond in the second residue, which explains why in Figure $5 \mathrm{C}$ and also in Figure $6 \mathrm{C}$ and $\mathrm{D}$ the deviations occur around the central amide bond. These additional conformations are probably not present in the experimental equilibrium.

For our understanding of the sampling of conformational space, it is also interesting to determine, how much the conformational spaces sampled by the various simulations of Val-Phe overlap. This is done by clustering the concatenated trajectories (structures taken every $20 \mathrm{ps}$ ) and analysing the composition of the clusters. ${ }^{[9,36]}$ The result is presented in Figure $8 \mathrm{~A}$. By far the largest cluster (25\% of all structures) represents the 10 -membered ring formed by $\mathrm{HB}_{10}$. This cluster only contains structures from simulations Val-Phe ${ }_{S S, 298}$ and Val-Phe ${ }_{S S, 340} . \mathrm{HB}_{10}$ is found in three other clusters (among the first 30 clusters): cluster 2 containing structures from Val-Phe ${ }_{S S, 298}, V_{\text {Val-Phe }}^{S S, 340}$ and Val-Phe ${ }_{S R, 340}$, cluster 12 again containing structures from Val-Phe $\mathrm{V}_{S, 298}$ and Val$\mathrm{Phe}_{S S, 340}$, and cluster 15 only containing structures from Val$\mathrm{Phe}_{S R, 298}$ and Val-Phe $\mathrm{S}_{S, 340}$. This shows, that the conformational space occupied by the two isomers is separated, even if one only considers the 10 -membered ring structures. Only few clusters contain structures from both the $S S$ and the $S R$

Figure 7. Torsional angle profiles in the MD simulations of the various $\beta$ dipeptides in methanol at different temperatures. A) Val-Phe at $298 \mathrm{~K}$; B) Val-Phe at $340 \mathrm{~K}$; C) di-Ala and di-Gly; grey filled curves: $S R$ isomer; striped dashed curves: $S S$ isomer; empty solid curves (only panel C): diGly. Definition of the torsional angles ( $i$ : residue number): $\mathrm{a}_{\mathrm{i}}: \mathrm{C}$ (carbon$\mathrm{yl})^{\mathrm{i}-1}-\mathrm{N}^{\mathrm{i}}-\mathrm{C}_{\beta}^{\mathrm{i}}-\mathrm{C}_{\alpha}^{\mathrm{i}} ; \mathrm{b}_{\mathrm{i}}: \mathrm{N}^{\mathrm{i}}-\mathrm{C}_{\beta}^{\mathrm{i}}-\mathrm{C}_{\alpha}^{\mathrm{i}}-\mathrm{C}(\text { carbonyl })^{\mathrm{i}} ; \mathrm{c}_{\mathrm{i}}: \mathrm{C}_{\beta}^{\mathrm{i}}-\mathrm{C}_{\alpha}^{\mathrm{i}}-\mathrm{C}(\text { carbonyl })^{\mathrm{i}}-\mathrm{N}^{\mathrm{i}+1} ; \mathrm{d}_{1}$ : $\mathrm{C}_{\beta}^{1}-\mathrm{C}_{\alpha}^{1}-\mathrm{C}_{\gamma}^{1}-\mathrm{C}_{\delta}^{1} ; \mathrm{d}_{2}: \mathrm{N}^{2}-\mathrm{C}_{\beta}^{2}-\mathrm{C}_{\gamma}^{2}-\mathrm{C}_{\delta}^{2}$.
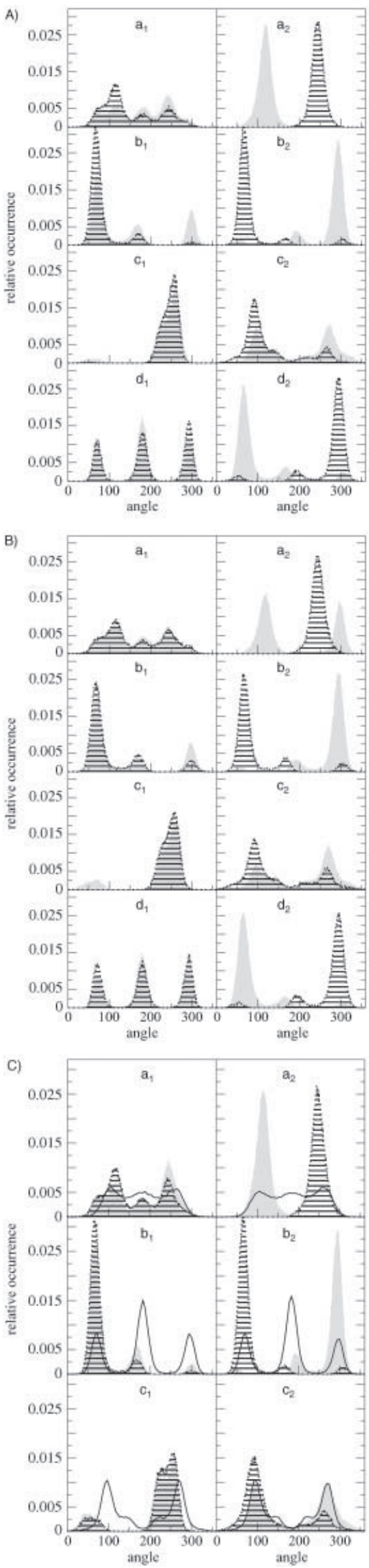

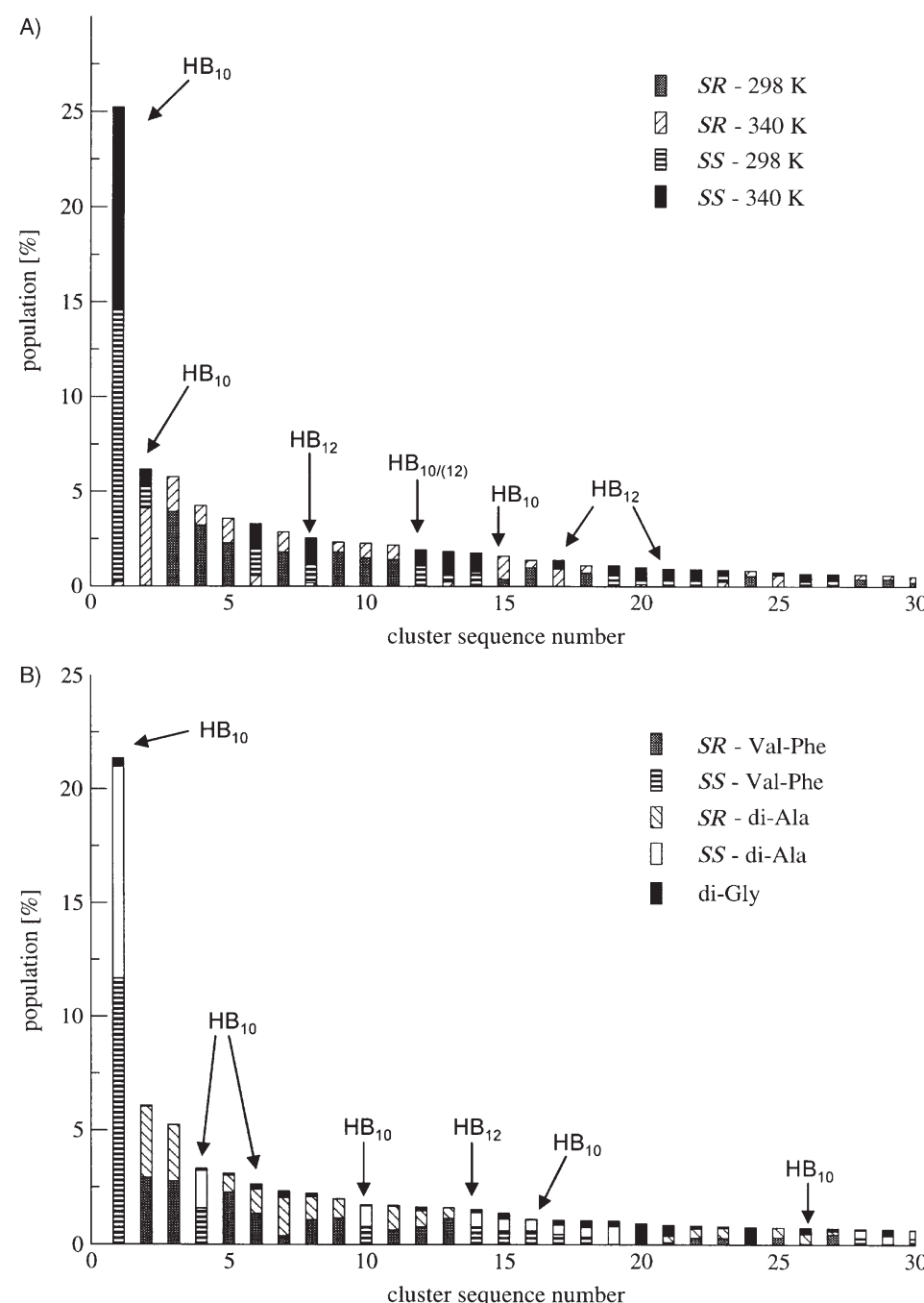

Figure 8. Clustering of merged trajectories: total cluster populations and fraction that belongs to the single MD trajectories. A) Concatenation of Val-Phe $_{S R, 298}$, Val-Phe ${ }_{S R, 340}$, Val-Phe VSS,298 $_{\text {and Val-Phe }}$ V $_{S, 340}$ (structures taken every $20 \mathrm{ps}, 7000$ structures in total); B) concatenation of Val-Phe $\mathrm{S}_{S R, 298}$, Val-Phe ${ }_{S S, 298}$, di-Ala ${ }_{S R, 298}$, di-Ala ${ }_{S S, 298}$ and di-Gly ${ }_{298}$ (structures taken every $20 \mathrm{ps}, 8750$ structures in total). Hydrogen bonds that are predominant in a cluster are indicated.

isomers, and overlap always involves simulation Val$\mathrm{Phe}_{S R, 340}$.

di-Ala $_{S R}$, di-Ala ${ }_{S S}$ and di-Gly-Influence of the side chains: How much is the backbone conformation dominated by the type and the bulkiness of the side chains? This can easily be investigated by performing simulations with less side chain atoms. Figures $2 \mathrm{E}-\mathrm{G}$ show the central member structures of the largest clusters of the di-alanine and di-glycine variants of the Val-Phe peptides. The predominant conformation of di-Ala $_{S S, 298}$ (Figure 2F) is a 10-membered ring, just as in Val$\mathrm{Phe}_{S S, 298}$ (Figure 2C). The predominant conformation of di$\mathrm{Ala}_{S R, 298}$ (Figure 2E) is an open, S-shaped structure, just as

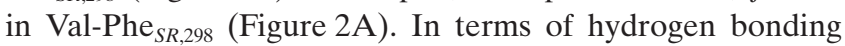
(Table 1), di-Ala ${ }_{S S, 298}$ lies between Val-Phe ${ }_{S S, 298}$ and Val$\mathrm{Phe}_{S S, 340}$, and di-Ala ${ }_{S R, 298}$ lies between Val-Phe ${ }_{S R, 298}$ and Val$\mathrm{Phe}_{S R, 340}$, respectively. This shows, that the type of side chain does not alter the conformational preferences of each isomer much, they are determined by the substitution pattern and the stereochemistry in the backbone. The fact that the properties of the di-Ala simulations at $298 \mathrm{~K}$ range somewhere between those of the Val-Phe simulations at 298 and at $340 \mathrm{~K}$ indicates, that reducing the side chains leads to enhanced flexibility. This is confirmed by Figure $3 \mathrm{E}$ and F, where several transitions between the open and the closed structures are observed for di-Ala. Also in case of the torsional angle profiles (see Figure 7), the di-Ala 298 simulations (Figure 7C) show features both of Val-Phe ${ }_{298}$ (Figure 7A) and of Val-Phe ${ }_{340}$ (Figure 7B), namely the additional rotamer $\left(b_{1}\right)$ around the $C_{\beta}-C_{\alpha}$ bond in residue 1 , which is only sampled by di-Ala ${ }_{S R, 298}$ (grey) not by di-Ala ${ }_{S S, 298}$ (striped), just as in Val-Phe ${ }_{298}$, and an additional rotamer (at $60^{\circ}, \mathrm{c}_{1}$ )

${ }^{0}$ around the $\mathrm{C}_{\alpha}-\mathrm{C}=\mathrm{O}$ bond in residue 1 which is sampled significantly only by di-Ala ${ }_{S S, 298}$, di-Ala ${ }_{S R, 298}$ and Val-Phe SR,340. $_{\text {. }}$ The first cluster of di-Gly ${ }_{298}$ is an extended conformation, and the low hydrogen-bond populations and the low cluster populations show that di-Gly is extremely flexible. The torsional angle profiles of di-Gly (Figure 7C, solid lines) are almost as perfectly symmetrical as expected, as no stereocenter induces any preferences in any direction, and di-Gly samples rotamers which di-Ala and Val-Phe cannot sample because of their side chains. Figure 8 (lower panel) shows the populations of the clusters of a combined clustering analysis of all five dipeptide simulations at $298 \mathrm{~K}$ (Val-

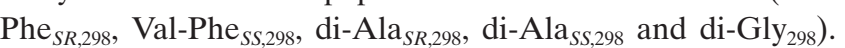
It shows, that at this temperature, the conformational space sampled by the two stereoisomers has no overlap, that is, clusters either contain structures from Val-Phe ${ }_{S S, 298}$ and di$\mathrm{Ala}_{S S, 298}$ or structures from Val-Phe ${ }_{S R, 298}$ and di-Ala ${ }_{S R, 298}$.

${ }^{3}$ Most of the clusters contain a tiny fraction of the di-Gly 298 simulation, which shows that di-Gly samples the complete space accessible to the substituted dimers. Additionally, there are pure di-Gly clusters, confirming the above observation that di-Gly samples more rotamers than the substituted dimers.

\section{Conclusion}

The MD simulations of the $S S$ and $S R$ diastereomers of the Val-Phe $\beta$-dipeptide at $298 \mathrm{~K}$ confirm the predicted structural differences. The $S S$ isomer forms a 10 -membered ring, whereas the $S R$ isomer prefers an S-shaped open conformation. As predicted for peptides where the amide bond is conformationally restricted by two adjacent side chains, the hydrogen bond which leads to a 12-membered ring structure is only very lowly populated. The MD simulations reproduce the available experimental data quite well. Upon closer investigation, it has to be questioned though, whether the experimental data can significantly distinguish between $S R$ and $S S$ isomer. As far as ${ }^{3} J$ coupling constants are concerned, the deviations between simulated and experimental values for each isomer are in the same order of magnitude as the deviations between the (structurally completely different) diastereomers. Yet, the simulations correctly predict which of the two isomers yields the larger ${ }^{3} J$ value. After a 
temperature increase in the simulations, which at least in case of the $S R$ isomer causes substantial structural changes, the agreement of the simulated and measured ${ }^{3} J$ values remains essentially unaffected. Similar conclusions can be drawn for the ${ }^{1} \mathrm{H}$ NMR ROESY spectra. Again, the differences in the spectral intensities between $S S$ and $S R$ are small, and it requires an extremely careful analysis of a rather small set of cross peaks to find the structural and dynamical differences between the isomers reflected in the spectrum. For such a detailed analysis, it is essential to replace the conventional method of comparing distance averages computed from the MD simulations with experimentally derived distances by a back-calculation of the relaxation rates and intensities from the MD simulations using the flexible approach which includes all aspects of internal dynamics. The differences in the spectra can be understood by investigating the torsional angle profiles of the backbone and the first side chain dihedral angles of the MD simulations. Increasing the temperature in the simulations leads in case of the $S R$ isomer of Val-Phe to enhanced sampling of dihedral space and to formation of new conformations. One observes the formation of both structures (10- and 12-membered rings) at $340 \mathrm{~K}$. The conformational study of the two Val-Phe isomers, their di-Ala and the di-Gly variants shows that the conformational space sampled by the di-Ala isomers is very similar to the space sampled by the corresponding Val-Phe isomers. The properties of di-Ala at $298 \mathrm{~K}$ lie between the properties of Val-Phe at $298 \mathrm{~K}$ and at $340 \mathrm{~K}$. Thus, variation of side chains does not completely alter the conformational preferences of each isomer, it mainly determines the flexibility of the molecule. The di-Gly simulation, which shows a high degree of flexibility, samples the complete space accessible to the substituted dimers and additional conformations which are accessible only to the unsubstituted peptide.

\section{Experimental Section}

The $\beta$-peptides were synthesized as previously described (ref. [3] and references therein).

NMR Spectroscopy of $\boldsymbol{\beta}$-peptides sample: $10-12 \mathrm{mg}$ dissolved in $0.6 \mathrm{~mL}$ $\mathrm{CD}_{3} \mathrm{OH}$. 1D NMR (DRX 500): ${ }^{1} \mathrm{H}$ NMR $(500 \mathrm{MHz}$ ): suppression of the $\mathrm{CD}_{3} \mathrm{OH}$ signal by presaturation; $90 \mathrm{~K}$ data points, 128 scans, $5.6 \mathrm{~s}$ acquisition time. $\left\{{ }^{1} \mathrm{H}\right\}$-BB-decoupled $-{ }^{13} \mathrm{C}$ NMR $(125 \mathrm{MHz})$ : $80 \mathrm{~K}$ data points, $20 \mathrm{~K}$ scans, $1.3 \mathrm{~s}$ acquisition time, $1 \mathrm{~s}$ relax. delay $45^{\circ}$ excitation pulse. Processed with $1.0 \mathrm{~Hz}$ exponential line broadening. 2D NMR: All with solvent suppression by presatd DQF-COSY $\left(500 \mathrm{MHz}, \mathrm{CD}_{3} \mathrm{OH}\right)$ with pulsed field gradients (PFG) for coherence pathway selection ${ }^{337}$ : Acquisition: $2 \mathrm{~K}\left(t_{2}\right) \times 512\left(t_{I}\right)$ data points. 10 scans per $t_{1}$ increment, $0.17 \mathrm{~s}$ acquisition time in $t_{2}$; relaxation delay $2.0 \mathrm{~s}$. TPPI quadrature detection in $\omega_{1}$. Processing: Zero filling and FT to $1 \mathrm{~K} \times 1 \mathrm{~K}$ real/real data points after multiplication with $\sin ^{2}$ filter shifted by $\pi / 3$ in $\omega_{2}$ and $\pi / 2$ in $\omega_{1}$. ROESY ${ }^{[38]}\left(500 \mathrm{MHz}, \mathrm{CD}_{3} \mathrm{OH}\right)$. Acquisition: five ROESY spectra with $t_{\mathrm{m}}=50,100,150,200$ and $400 \mathrm{~ms}$ were acquired. CW-spin lock $(2.7 \mathrm{kHz})$ between trim pulses, $2 \mathrm{~K}\left(t_{2}\right) \times 512\left(t_{1}\right)$ data points, 64 scans per $t_{1}$ increment. $0.17 \mathrm{~s}$ acquisition time in $t_{2}$, other parameters identical to DQFCOSY. Processing: Zero filling and FT to $1 \mathrm{~K} \times 512 \mathrm{~K}$ real/real data data points points after multiplication by $\cos ^{2}$ filter in $\omega_{2}$ and $\omega_{1}$. Baseline correction with 3 rd degree polynomial in both dimensions. Crosspeak intensities were determined via integration of the peak volumes with XWINNMR. For comparison with the MD simulations, a reduced set of 11 unambiguous, reliable peaks from proton pairs involving $\mathrm{N}-\mathrm{H}, \mathrm{C}_{\alpha}-\mathrm{H}$,
$\mathrm{C}_{\beta}-\mathrm{H}$, and $\mathrm{C}_{\gamma}-\mathrm{H}$ was considered. No indications of aggregation were observed.

Computational methods: All simulations were performed using the GROMOS96 package of programs ${ }^{[28,29]}$ with the standard GROMOS96 43A1 united atom force field. ${ }^{[2]}$ The methanol model was taken from the standard GROMOS96 set of solvents. ${ }^{[28,29,39]}$ Initially, the solute was placed in an extended conformation (with all backbone torsional angles in trans) at the center of a truncated octahedron with a minimum distance of the solute atoms to the square box walls of $1.8 \mathrm{~nm}$. The resulting numbers of solvent molecules are 886 (Val-Phe ${ }_{S R, 298}$ and Val-Phe ${ }_{S R, 340}$ ), 888 (Val-Phe ${ }_{S S, 298}$ and Val-Phe $\left.{ }_{S S, 340}\right), 894\left(\right.$ di-Ala $\left._{S S}\right), 889\left(\right.$ di-Ala $\left._{S R}\right), 893$ (di-Gly). Periodic boundary conditions were applied. The system was relaxed by initially performing a steepest descent energy minimization. The MD simulations were started by taking the initial velocities from a Maxwellian distribution at the corresponding temperature. Solvent and solute were independently weakly coupled to a temperature bath with a relaxation time of $0.1 \mathrm{ps} .{ }^{[40]}$ The pressure was calculated with a molecular virial and kept constant at $1 \mathrm{~atm}$ by also applying the weak coupling algorithm with a relaxation time of $0.5 \mathrm{ps}$ and an isothermal compressibility of $4.575 \times 10^{-4}\left(\mathrm{~kJ} \mathrm{~mol}^{-1} \mathrm{~nm}^{-3}\right)^{-1}$. Bond lengths were constrained using the SHAKE algorithm with a geometric tolerance of $10^{-4},{ }^{[41]}$ so that the time step for the leapfrog integration scheme could be set to $0.002 \mathrm{ps}$. For the non-bonded interactions a twin-range method with cutoff radii of 0.8 and $1.4 \mathrm{~nm}$ was used. ${ }^{[28,29]}$ Outside the longer cutoff radius a reaction field approximation $^{[42]}$ was used with a relative dielectric permittivity of 17.7. The center of mass motion of the whole system was removed every 10000 time steps. The system was equilibrated for $5 \mathrm{~ns}$ and the trajectory coordinates were saved every $0.5 \mathrm{ps}$ for analysis over a period of $35 \mathrm{~ns}$.

Structure analysis: A cluster analysis was performed on all trajectories using the structures every at $0.01 \mathrm{~ns}$. Clustering was done as described in ref. [30] by performing a rotational and translational atom-positional least-squares fit on every pair of structures and calculating the atom-positional root-mean-square difference (RMSD) between the two structures using all backbone atoms. The similarity criterion used was an RMSD $\leq$ $0.05 \mathrm{~nm}$. The criterion for a hydrogen bond in a given structure was a maximum distance of $0.25 \mathrm{~nm}$ between the hydrogen atom and the acceptor atom and a minimum angle of $135^{\circ}$ between donor, hydrogen and acceptor.

NMR analysis: The method to back-calculate NOESY and ROESY spectra based on MD simulations can be found in ref. [26]. Compared to the procedure described there a small modification was made, namely the spectral density functions were computed for all proton pairs and not only for a selected set. This was made possible by the use of fast Fourier transforms (FFTs) when computing the time-correlation functions. ${ }^{3} J$ coupling constants were calculated from the MD simulations using the Karplus relation ${ }^{[43]}$

${ }^{3} J_{\mathrm{HH}}=A \cos ^{2} \theta+B \cos \theta+C$

in which $\theta$ is the dihedral angle defined by the three bonds and the two protons. The parameters $A, B$ and $C$ were taken from the literature as summarized in Table 4.

Table 4. Coefficients of the Karplus equation ${ }^{[43]}$ [Eq. (1)]

\begin{tabular}{lllll}
\hline Type & $A$ & $B$ & $C$ & Ref. \\
\hline${ }^{3} J_{\mathrm{H}^{\mathrm{N}} \mathrm{H}^{\mathrm{C}}}$ & 6.4 & -1.4 & 1.9 & {$[32]$} \\
& 9.5 & -1.4 & 0.3 & {$[33]$} \\
& 7.0 & -1.4 & 1.7 & {$[34]$} \\
${ }^{3} J_{\mathrm{H}^{\mathrm{C}} \mathrm{H}^{\mathrm{C}}}$ & 7.9 & -1.1 & 0.7 & {$[35]$} \\
\hline
\end{tabular}

\section{Acknowledgement}

Financial support was obtained from the Swiss National Science Foundation, project number 2000-063590.00, and through the National Center of 
Competence in Research (NCCR) Structural Biology of the Swiss National Science Foundation which is gratefully acknowledged.

[1] D. J. Hill, M. J. Mio, R. B. Prince, T. S. Hughes, J. S. Moore, Chem. Rev. 2001, 101, 3893-4012.

[2] R. P. Cheng, S. H. Gellman, W. F. DeGrado, Chem. Rev. 2001, 101, 3219-3232.

[3] M. Rueping, J. V. Schreiber, G. Lelais, B. Jaun, D. Seebach, Helv. Chim. Acta 2002, 85, 2577-2593.

[4] W. F. van Gunsteren, R. Bürgi, C. Peter, X. Daura, Angew. Chem. 2001, 113, 363-367; Angew. Chem. Int. Ed. 2001, 40, 351-355.

[5] X. Daura, B. Jaun, D. Seebach, W. F. van Gunsteren, A. E. Mark, J. Mol. Biol. 1998, 280, 925-932.

[6] X. Daura, K. Gademann, B. Jaun, D. Seebach, W. F. van Gunsteren, A. E. Mark, Angew. Chem. 1999, 111, 249-253; Angew. Chem. Int. Ed. 1999, 38, 236-240;

[7] X. Daura, K. Gademann, H. Schäfer, B. Jaun, D. Seebach, W. F. van Gunsteren, J. Am. Chem. Soc. 2001, 123, 2393-2404.

[8] X. Daura, I. Antes, W. F. van Gunsteren, W. Thiel, A. E. Mark, Proteins 1999, 36, 542-555.

[9] A. Glättli, X. Daura, D. Seebach, W. F. van Gunsteren, J. Am. Chem. Soc. 2002, 124, 12972-12978.

[10] D. Korzhnev, M. Billeter, A. Arseniev, V. Orekhov, Prog. Nucl. Magn. Reson. Spectrosc. 2001, 38, 197-266.

[11] M. W. F. Fischer, A. Majumdar, E. R. P. Zuiderweg, Prog. Nucl. Magn. Reson. Spectrosc. 1998, 33, 207-272.

[12] G. Lipari, A. Szabo, J. Am. Chem. Soc. 1982, 104, 4546-4559.

[13] J. W. Peng, G. Wagner, J. Magn. Reson. 1992, 98, 308-332.

[14] D. E. Woessner, J. Chem. Phys. 1962, 36, 1-4.

[15] V. A. Daragan, K. H. Mayo, Prog. Nucl. Magn. Reson. Spectrosc. 1997, 31, 63-105.

[16] D. Neuhaus, M. Williamson, The Nuclear Overhauser Effect in Structural and Conformational Analysis, Wiley-VCH, New York, 2000.

[17] S. Macura, R. R. Ernst, Mol. Phys. 1980, 41, 95-117.

[18] J. W. Keepers, T. L. James, J. Magn. Reson. 1984, 57, 404-426.

[19] R. Boelens, T. M. G. Koning, G. A. van der Marel, J. H. van Boom, R. Kaptein, J. Magn. Reson. 1989, 82, 290-308.

[20] A. Kumar, R. R. Ernst, K. Wüthrich, Biochem. Biophys. Res. Commun. 1980, 95, 1-6.

[21] J. Tropp, J. chem.. Phys. 1980, 72, 6035-6043.

[22] A. G. Palmer III, D. A. Case, J. Am. Chem. Soc. 1992, 114, 90599067
[23] T. Schneider, A. Brünger, M. Nilges, J. Mol. Biol. 1999, 285, $727-$ 740.

[24] S. E. Feller, D. Huster, K. Gawrisch, J. Am. Chem. Soc. 1999, 121, $8963-8964$

[25] J. J. Prompers, R. Brüschweiler, J. Am. Chem. Soc. 2002, 124, 45224534.

[26] C. Peter, X. Daura, W. F. van Gunsteren, J. Biomol. NMR 2001, 20, 297-310.

[27] K. A. Feenstra, C. Peter, R. M. Scheek, W. F. van Gunsteren, A. E. Mark, J. Biomol. NMR 2002, 23, 181-194.

[28] W. R. P. Scott, P. H. Hünenberger, I. G. Tironi, A. E. Mark, S. R. Billeter, J. Fennen, A. E. Torda, T. Huber, P. Krüger, W. F. van Gunsteren, J. Phys. Chem. A 1999, 103, 3596-3607.

[29] W. F. van Gunsteren, S. R. Billeter, A. A. Eising, P. H. Hünenberger, P. Krüger, A. E. Mark, W. R. P. Scott, I. G. Tironi, Biomolecular Simulation: The GROMOS96 Manual and User Guide, Vdf Hochschulverlag AG an der ETH Zürich, Zürich, 1996.

[30] X. Daura, W. F. van Gunsteren, A. E. Mark, Proteins 1999, 34, $269-$ 280

[31] A. de Marco, M. Llinas, K. Wüthrich, Biopolymers 1978, 17, 617636.

[32] A. Pardi, M. Billeter, K. Wüthrich, J. Mol. Biol. 1984, 180, 741-751.

[33] R. Brüschweiler, D. Case, J. Am. Chem. Soc. 1994, 116, 1119911200

[34] A. C. Wang, A. Bax, J. Am. Chem. Soc. 1996, 118, 2483-2494.

[35] J. M. Schmidt, M. Blümel, F. Löhr, H. Rüterjans, J. Biomol. NMR 1999, 14, 1-12.

[36] R. Baron, D. Bakowies, W. F. van Gunsteren, X. Daura, Helv. Chim. Acta 2002, 85, 3872-3882.

[37] A. L. Davis, E. D. Laue, J. Keeler, D. Moskau, J. Lohman, J. Magn. Reson. 1991, 94, 637-644.

[38] C. Griesinger, R. R. Ernst, J. Magn. Reson. 1987, 75, 261-271.

[39] R. Walser, A. E. Mark, W. F. van Gunsteren, M. Lauterbach, G. Wipff, J. Chem. Phys. 2000, 112, 10450-10459.

[40] H. J. C. Berendsen, J. P. M. Postma, W. F. van Gunsteren, A. DiNola, J. R. Haak, J. Chem. Phys. 1984, 81, 3684-3690.

[41] J.-P. Ryckaert, G. Ciccotti, H. J. C. Berendsen, J. Comput. Phys. 1977, 23, 327-341.

[42] I. G. Tironi, R. Sperb, P. E. Smith, W. F. van Gunsteren, J. Chem. Phys. 1995, 102, 5451-5459.

[43] M. Karplus, J. Chem. Phys. 1959, 30, 11-15. 